\title{
Monitoring eye movements of slow and fast learners'
}

\author{
E. J. HALTRECHT ${ }^{2}$ AND P. D. MCCORMACK \\ CARLETON UNIVERSITY, OTTAWA, CANADA
}

Eye Movements of slow and fast learners were monitored as they studied a 7-item P-A list. The findings were consistent with a two-stage interpretation of verbal leaming. The slow learners appeared to take longer in Stage 1, a relatively brief process, and to experience considerable difficulty in Stage 2.

In each of two earlier investigations (McCormack \& Haltrecht, 1966; McCormack, Haltrecht, \& Hannah, 1966a) eye movements were monitored while Ss learned verbal paired-associates (P-A) by the recall method. During early learning trials, they viewed the response out of proportion to the stimulus while looking first to the stimulus and then to the response. As learning progressed, the stimulus came to be viewed out of proportion to the response, with Ss exhibiting a pattern of looking first to the stimulus then to the response and then back to the stimulus. These findings were interpreted as being consistent with a two-stage conceptualization of verbal learning (Underwood \& Schulz, 1960), Ss presumably consolidating the responses during the initial or "response-learning" phase and then connecting them to the appropriate stimuli during the second or "hook-up" stage. These data, and others (McCormack, Haltrecht, \& Hannah, 1966b), were also consistent with the notion that the two stages overlap, with the greatest amount of response-consolidation occurring within the first few trials. The purpose of the present study was to examine the eye movements of slow and fast learners in an attempt to reveal additional information regarding the two-stage conceptualization.

\section{Subjects and Apparatus}

Ss were 51 male and 49 female first and second year university students. Lists were made of seven pairs of CVCs, with $\mathrm{m}^{\prime}$ values (Noble, 1961) within the range $1.80-1.93$, and were projected by means of a timercontrolled Carousel unit to a green -surfaced chalkboard $1.4 \mathrm{~m}$ from $\mathrm{S}$ and $2.4 \mathrm{~m}$ from the projector. Eye movements were recorded, for slow and fast Ss only, by means of a head-mounted eye-marker camera (Mackworth \& Thomas, 1962), consisting of a $2 \times 8 \mathrm{~mm}$ motion picture unit filming at a speed of 16 frames per sec. and a periscope that transmits a spot of light from the cornea to the film. The film, when developed, shows the P-A slides and, superimposed on each, a bright circular spot indicating the approximate position of actual fixation. The data were analyzed frame by frame by examining the S-R slides through a film-viewer. Procedure

The 100 Ss were required to learn a list which was devised for the purpose of establishing two extreme groups of 14 Ss each, Group S (slow) and Group F (fast). On any given trial, the seven S-R pairs were presented consecutively. During this study phase, $S$ was instructed to examine the pairs in an attempt to learn them. Following a 2-sec. interval, the seven stimuli were presented, with $S$ attempting to recall the appropriate responses during this test phase. Slides changed every 2 sec., the intertrial interval was 2 sec.s and a 1-min. rest was given at the end of every four trials. The list was presented in each of four different random orders in an attempt to minimize serial learning and the session was terminated when a criterion of one errorless trial was attained. Seven Ss showed little indication of reaching criterion and so were stopped at the end of 24 trials. Eye movements were not recorded during this screening session.

The 16 fastest and 24 slowest Ss were later required to learn a second list under conditions similar to those of the screening task. Eye movements were recorded during the first 16 trials and the session was terminated at that point unless Ss had not reached criterion, in which case additional trials were provided until one without any errors was recorded. Of the fast group, two Ss were not consistently fast from list to list and so were discarded, which left 14 Ss in Group F. Nine Ss of the slow group could not be considered consistently slow because of their performance on the second list. This, in addition to the spoilage of film of one other $S$, left 14 in Group $S$.

\section{Results and Discussion}

The number of trials required to reach criterion on lists 1 and 2 for Ss of Group S were as follows, with an asterisk indicating those whose first session was terminated following the 24 th trial: $22-38, * 24-29, * 24-30$, *24-31, *24-32, *24-32, *24-48, 25-36, 25-53, 27-37, 28-28, 31-40, 31-50, 34-23. Comparable results for Group $F$ were as follows: 4-7, 5-8, 6-3, 6-9, 7-6, 7-7, 8-7, 8-8, 9-4, 9-6, 10-5, 10-11, 11-7. Examination of these data reveals no doubt that extreme groups were successfully established.

The main fixation findings are summarized in Fig. 1. The first eight trials, in blocks of two, are shown along the abscissa while the ordinate represents mean number of frames per syllable-pair for those slides where $S$ and $R$ appeared together on the second list. The upper pair of functions represent Group $S$ frames while the lower pair are those of Group F. Broken curves indicate response frames while solid curves depict stimulus frames. The findings portrayed by Fig. 1 were examined statistically. A dependable Trials by Conditions (S vs. R) interaction was revealed $(p<.01)$, indicating in- 


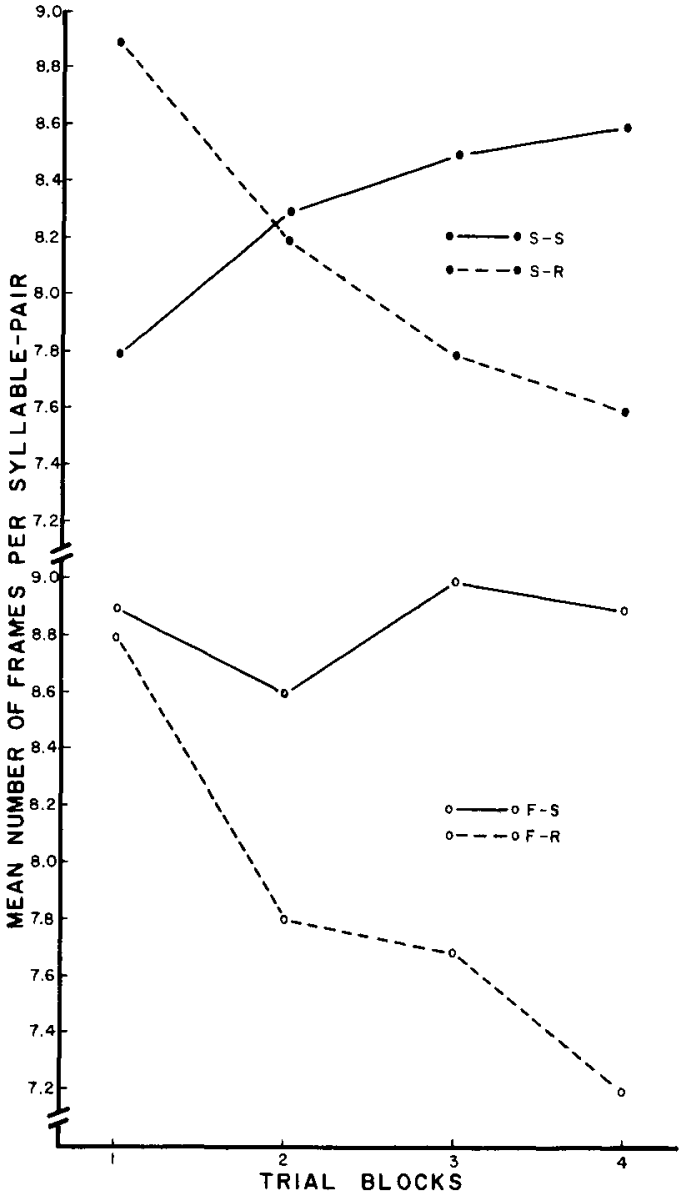

Fig. 1. Mean number of stimulus and response frames per syllablepair as a function of the first 8 trials, in blocks of 2 , for Groups $S$ and $\mathbf{F}$.

creased viewing with trials of the stimulus in relation to the response on the part of both groups of Ss, a finding consistent with previous studies. This analysis was supplemented by recording for each $\mathrm{S}$ that trial where the stimulus was first viewed out of proportion to the response. The mean for Group $S$ was reliably larger than that for Group $F(p<.025)$, as might be expected from examination of Fig. 1 which indicates that Group $F$ viewed the stimulus out of proportion to the response from the outset. It thus appears that, of the two groups, Group S has greater relative difficulty with response-consolidation. These Ss, however, appear to successfully complete Stage 1 and then encounter considerable difficulty in Stage 2. That they complete Stage 1 is borne out by the relatively early cross-over of their fixation functions as well as by intrusions of already-consolidated responses during early learning trials. The analysis of the data portrayed by Fig. 1 also revealed a statistically dependable Trials effect $(p<$ .001 ) as well as a reliable Groups by Trials interaction $(p<.025)$, indicating that $S s$ tend to look away from learned pairs, those of Group $F$ exhibiting this to a greater degree.

The three easiest and three most difficult CVC pairs were determined for each $S$ in Group $S$. When the two sets of fixation functions were examined, the cross-over point for the difficult pairs was observed to occur on a later trial than that for the easy pairs, a finding consistent with the notion that Stage 1 should be prolonged for difficult materials. Even with the difficult pairs, however, disproportionate stimulus-viewing became stable by the sixth trial, again indicating that Group $\mathrm{S}$ has its greatest difficulty in Stage 2. Eye movements were also examined, Group F showing S-R-S patterns from the outset while Group $S$ exhibited S-R patterns during the early trials and then later switched to S-R-S viewing. An analysis of these data, with mean number of eye movements plotted as a function of trials for both groups, revealed a statistically dependable Trials by Groups interaction $(p<.05)$. These observations are once more consistent with the notion that, of the two groups, Group S has greater relative difficulty in Stage 1 , but that it encounters its greater challenge in Stage 2.

The fixation functions and eye movement data of the slow Ss of the present study do not differ appreciably from those observed in previous investigations where the majority of Ss could be termed "average" learners. Their learning performance, however, is considerably different, this being presumably due to difficulties encountered in Stage 2. Thus visual monitoring techniques are unable to reveal the nature of the difficulty experienced by slow learners in Stage 2. Also, because of the apparent brevity of Stage 1 , it seems unlikely that these techniques will reflect any Stage 1 differences that might exist between groups of Ss, or between different types of materials, except at the extremities of these dimensions.

\section{References}

Mackworth, N. H.; \& Thomas, E. L. Head-mounted eye-marker camera. J. Opt. Soc. Amer., 1962, 52, 713-716.

McCormack, P. D., \& Haltrecht, E. J. Monitoring eye movements under two conditions of presentation of paired-associate materials. Canad. J. Psychol., 1966, 20, 154-159.

McCormack, P. D., Haltrecht, E. J., \& Hannah, T. E. Supplementary Report: Monitoring eye movements during the learning of successive paired-associate lists. J. verbal Learn. verbal Behav., $1966 \mathrm{a}$, in press.

McComack, P. D., Haltrecht, E. J., \& Hannah, T. E. Monitoring eye movements in two non-learning situations. Psychon. Sci., $1966 \mathrm{~b}, 6$, in press.

Noble, C. E. Measurement of associative value $(a)$, rated associations $\left(a^{\prime}\right)$ and scaled meaningfulness $\left(m^{\prime}\right)$ for the $2100 \mathrm{CVC}$ combinations of the English alphabet. Psychol. Rep., 1961, 8, 487521.

Underwood, B. J., \& Schulz, R. W. Meaningfulness and verbal learning. Chicago: Lippincott, 1960.

\section{Notes}

1. This research was supported by a grant-in-aid from the Associate Committee on Experimental Psychology of the National Research Council of Canada (Grant APA-78) and represents an M. A. thesis on the part of the senior investigator.

2. Now at York University, Toronto, Canada. 